\title{
Electromyographical Studies on Graves' Disease
}

\author{
By
}

\section{Kiyoshi Kawamura}

(川 村 唛)

\author{
From the Surgical Clinic of Prof. S-T. Katsura, \\ Tohoku University, Sendai
}

(Received for publication, May 12, 1954)

Recently, the study of EMG has made a considerable advance and its clinical application has become widely applied. The action potential of neuromuscular disorders of exophthalmic goiter has been studied.

\section{EXPERIMENTAL}

\section{Method}

The action current of motor units from $\mathrm{M}$. biceps brachii using a concentric needle electrode made by Adrian ${ }^{1 \prime}$ with an oscillograph equipped with a R.C. 4 stage amplifier was recorded.

Usually, normal distribution of spike discharge of human motor units is at random according to Tokizane ${ }^{2}$ and do not show any tendency of synchronization or grouping. However, as Denny Brown ${ }^{3 /}$ pointed out already, this distribution is influenced with various conditions, such as instability, fatigue, abnormal sensation, pain and cold weather; and this spike discharge is apt to show the tendency of grouping. In order to avoid the above mentioned phenomienon, the experiment was carried out under the following condition.

1) The patient was told to relax.

2) The test was performed in the early morning when the patient was not tired.

3) $1 \%$ novocaine was injected into the skin and fascia to avoid pain. When pain was complained, the needle was reapplied.

4) The patient was confirmed to have no loss of sensation or painful disease.

According to the results which were obtained, even though these conditions were kept in mind, the distribution of spike discharge in a normal person was considered to show a little tendency of grouping, while the action potentials of epileptics without any medical treatment were thought to have scattered spike distribution; therefore the action potential of 
epileptics was compared with that of the Graves' disease.

\section{Results}

1) In 18 cases of Graves' disease, grouping was observed in EMG according to the strength of tremor. Even in those patients with mild toxic symptoms who didn't show any tremor clinically, the tendency of grouping was observed. The appearance of this kind of grouping was different from that of Parkinson's disease in the following points.

In Graves' disease, grouping appeared only during voluntary contraction, while any abnormal action potential including grouping was not observed at rest. General skeletal musclc also showed grouping during voluntary contraction. However, as already being pointed out by Bishop ${ }^{4)}$ grouping which was observed in Parkinson's disease was also recorded during involuntary contraction.

When the EMG of antagonistic muscle are recorded simultaneously, the grouping of Graves' disease is synchronous, while that of Parkinson's disease is antagonistic.

In Parkinson's disease, the duration of grouping, the number of the impulses in one group, and the time course of the interval of groups are definite, while they are indefinite in Grave's disease. As the grouping in Graves' disease appears only during voluntary contraction, this character of grouping changes in accordance with the difference of contractions. Besides, in case of Graves' disease with mild toxic symptorns, the formation of grouping is imcomplete and the boundary is often difficult to decide.

2) The relationship of spike discharges of different motor units of which each group consists.

Only 2 or 3 motor units which constitute each group were recorded separately with a concentric needle electrode, and the relationship of spike discharge of different motor units was observed.

The more toxic symptoms were intense, the more spike discharges of different motor units approximated with each other. Finally, a complete grouping was composed. Therefore, in Graves' disease, as like as in Parkinson's disease, the spike discharge of each group was formed by that of different motor units. Could this tendency of approach of spike discharges from different motor units be shown in figures, it would be much more convenient to know the toxic degree of neuromuscular system in Graves' disease. An attempt was made to propose a hypothetic form for this purpose. The statistical method was used, and the following results were obtained.

3) The relationship of the degree of approximation of spike discharge of different motor unit and the severity of toxic conditions. 
a

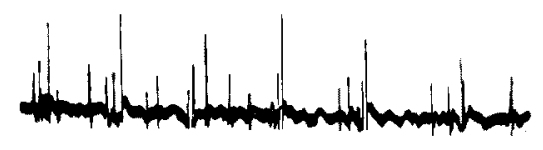

$\mathrm{b}$

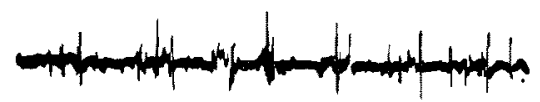

c

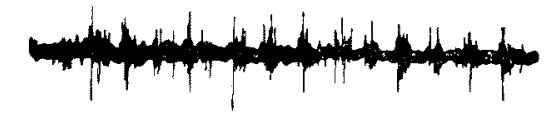

Fig. 1. EMG of M. biceps brachii from a patient of Graves' disease during voluntary contraction. a. Case with slight symptoms (tremor $(-)$ ): No tendency of grouping. b. Case with moderate symptoms (Tremor $(+))$ : Tendency of grouping. c. Case with severe symptoms (tremor (H)) : remarkalle fermation of grouping.

In order to measure the approach of spike discharge of different motor unit, appropriate method for analysis was unknown. Therefore, the following statistical method was applied and a hypothetic model is proposed. Fig. 2 shows its model. P unit discharges with $a$ impulse interval, and $D$ unit discharges with $b$ impulse interval. $a \& b$ take any value. $P \&$

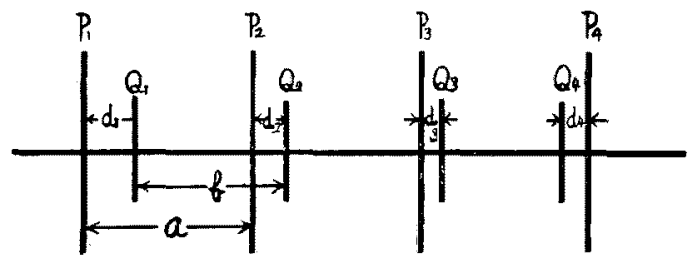

Fig. 2

$Q$ have no correlation between each other. They discharge independently. When spike discharge ( $P$ ) with a large impulse interval (a) is taken for a standard, $d$ is designated as the distance between the spike discharge $Q$ which is discharging closest to $\mathrm{P}$ and the spike discharge $\mathrm{P}$. In this case, it does not matter whether the spike discharges $Q$ locates in front or in the rear of $P$. Anyhow, the closest discharge to $P$ is taken. In order to read the approximation of $(P \& Q)$ regardless its relation to $P$, the absolute value is taken and designated as $d$. The arithmetic mean of $d$ is designated as the mean approach $(\bar{d})$. $\bar{d}$ of the EMG of 21 genuine epileptics who show scattered discharge pattern was found to take $1 / 4$ interval mean, i.e., 
a constant $b / 4$. This statistical form is calculated from Weyl's theory, the error area of the interval variation of each motor unit can be easily calculated, because the histogram of the interval shows a resembling Gaussian curve. In case of Graves' disease, the mean $\bar{d}$ of approach $d$ is smaller than $\mathrm{b} / 4$; and when the difference between $\bar{d}$ and $\mathrm{b} / 4$ is expressed in percentage of $\mathrm{b} / 4$, the approach of spike discharges from different motor units is $10-15 \%$ in less severe cases, $20 \%$ in mild cases, $35 \%$ in severe cases.

4) The fluctuation of the impulse interval of single motor unit. Fig. 3 shows the fluctuation by the interval diagram, and Fig. 3a shows less approach of the spike discharge of different motor unit in epileptics; the luctuation is small. Fig. 3b shows the EMG of Graves' disease with mild toxic symptoms. Fig. 3c shows that of Graves' disease with severe toxic symptoms. Fig. 3b, c both show irregular and rapid fluctuation according to the classification by Hagiwara ${ }^{5}$. Its tendency is more remarkable in the severe cases than in the others. The standard deviation of this irregular and rapid interval variation was calculated. Its relationship with mean impulse interval is shown in Fig. 4. In epileptics, the

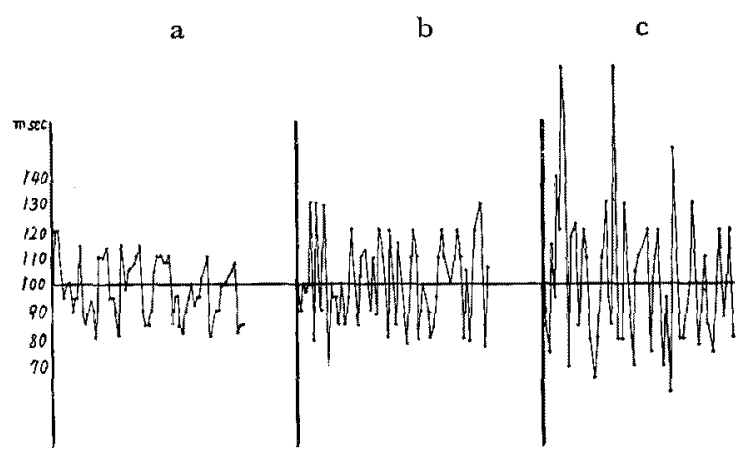

Fig. 3. The time course of the interval variation of a single motor unit. a. Epileptics. b. Graves' disease with slight symptoms. c. A severe case of Graves' disease.

fluctuation increases with the lentgh of mean impulse interval; and it always makes a definite curve. Contrary to epileptics, in Graves' disease, the fluctuation increases more intensely with the length of mean impulse interval than in epileptics. When it is classified in 3 groups according to the strength of grouping, 3 curves are written. This means that an increase of fluctuation of interval is rapid comparing with the slight increase of mean interval, so that clinically a long impulse interval is difficult to obtain. In other words, mean impulse interval becomes shorter. This is coincident with the findings by Sanderson ${ }^{6}$. The increase of fluctuation of the impulse interval is compared with the clinical symptoms. 


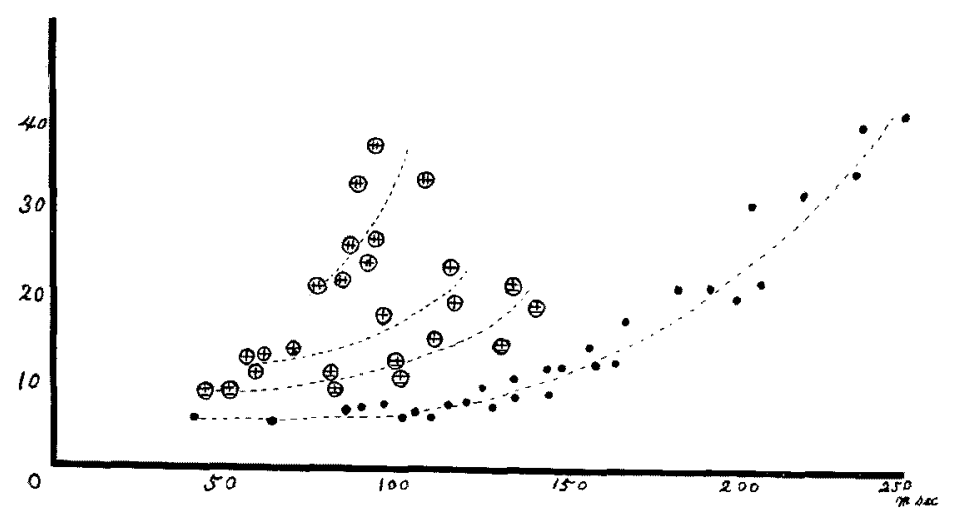

Fig. 4. Relationship between the standard deviation of interval variation of a single motor unit (abscissa) and the mean interval (ordinate). $\#$ Remarkable grouping in severe case of Graves' disease (percentage of the mean approach to normal: $35 \%$ ). + Tendency of grouping in moderate degree of Graves' disease (percentage of the mean approach to normal: 20\%). \pm No tendency of grouping in slight case of Graves' disease (percentage of the mean approach to normal: under 10\%). Genuine epileptics (percentage of the mean approach to normal: $0 \%$ ).

The relationship of the standard deviation of the fluctuation of interval of a single motor unit (A) with clinical symptoms (B).

In cases of high basal metabolism, the relationship is shown in Fig. 5. The increase of $\mathrm{A}$ and $\mathrm{B}$ is parallel. In this figure, standard deviation of fluctuation is influenced by the mean impulse interval. In order to clarify this changes, mark,++ and \pm are used. As being explained previously, both relationship is shown in Fig. 4.

The relationship between the standard deviation and the increase or instability of pulse is shown in Fig. 6 and Fig. 7, respectively. Those which show large fluctuation of impulse interval are accompanied with both increase and instability of pulse.

5) The relationship of the fluctuation of impulse interval of a single motor unit with correlation of two motor units. As being explained in the above paragraph about the increase of interval variation of a single motor unit in case of the tendency of grouping, it is exemplified in the following paragraph.

In Fig. 8, the relationship of approach percentage of spike discharge of two motor units taken by the oscillograph with the value of fluctuation of impulse interval of each unit calculated by standard deviation is shown. Two motor units that were calculated from mean approach were connected by the dotted lines. The more the approach is remarkable, the more the fluctuation of impulse interval of single motor units increases. 
Eig. 5

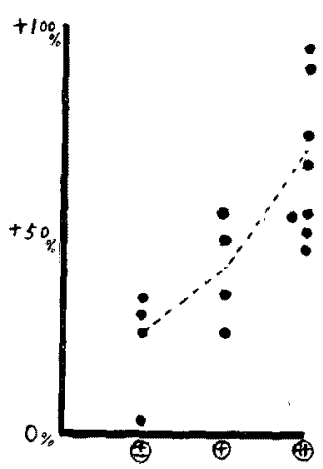

Fig. 6

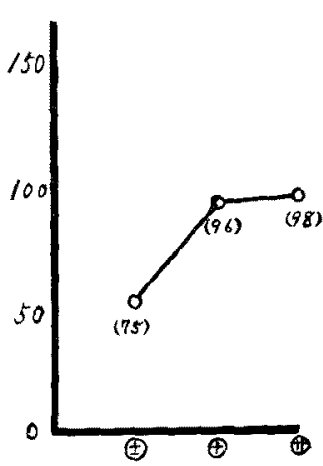

Fig. 7

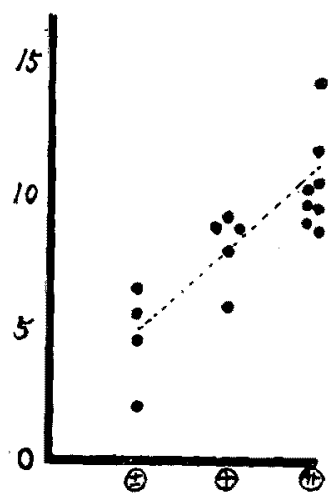

Fig. 5. Relationship between hypermetabolism (abscissa) and the standard deviation of the interval variation dotted line connects the mean value (ordinate).

Fig. 6. Relationship between pulsation per min. (abscissa) and standard deviation of interval variation of a single motor unit (ordinate).

Fig. 7. Relationship between standard deviation of pulse fluctuation (abscissa) and standard deviation of impulse fluctuation (ordinate).

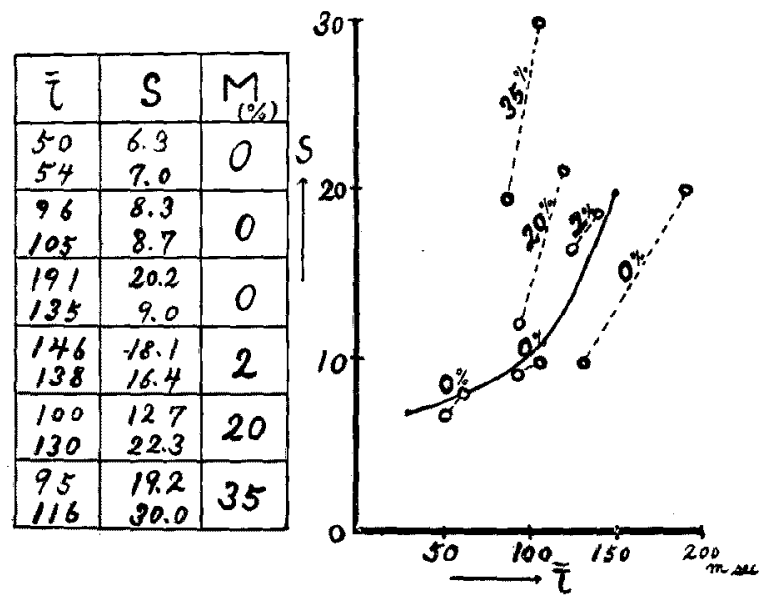

Fig. 8. Showing the standard deviation of interval variation of a single motor unit (S), the percentage of mean approach to normal between two motor units $(\mathrm{M})$ and the mean interval $(\tilde{\tau})$.

\section{SUMMARY}

The characteristics of the EMG of Graves' disease is that the distribution of spike discharge becomes more irregular than normal during voluntary muscle contraction. This irregularity is due to the following 3 factors.

1. The spike discharges of different motor units are apt to approach. 
2. The fluctuation of impulse interval of single motor unit becomes remarkable.

3. Mean impulse interval becomes shorter.

This phenomenon shows a tendency of increase or decrease with toxic symptoms, i.e., high basal metabolism, tachycardia and the instability of the pulse.

It is still unknown how this fluctuation occurs in what part of the motor unit. Further study is required.

\section{References}

1) Adrian \& Bronk, J. Physiol., 1929, 67, 119.

2) Tokizane \& Tsuyama, Kindenzu No Rinsho (Jap.), 1952, 63.

3) Denny-Brown, Arch. Neurol. Psychiat., 1950, 64, 141.

4) Bishop, Clare \& Price, J. Appl. Physiol., 1948, 1, 124.

5) Hagiwara, Ritsuchi Shizenkagaku Kenkyujo Hokoku (Jap.). 1943, 3, 19 ; Hagiwara \& Wakabayashi, No Kenkyu (Jap.), 1949, 5, 43.

6) Sanderson, Med. J. Australia, 1949, 1, 800. 